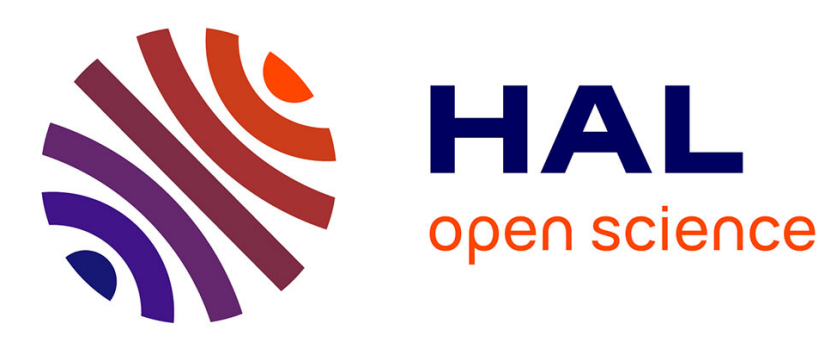

\title{
Numerical Instability of Classical Free-Interface Component Mode Synthesis Techniques
}

\author{
Ronnie Bladh, Christophe Pierre, Matthew P Castanier
}

\section{To cite this version:}

Ronnie Bladh, Christophe Pierre, Matthew P Castanier. Numerical Instability of Classical FreeInterface Component Mode Synthesis Techniques. AIAA Journal, 2003, 41 (8), pp.1621-1624. 10.2514/2.2123 . hal-01711566

\section{HAL Id: hal-01711566 \\ https://hal.science/hal-01711566}

Submitted on 27 Mar 2018

HAL is a multi-disciplinary open access archive for the deposit and dissemination of scientific research documents, whether they are published or not. The documents may come from teaching and research institutions in France or abroad, or from public or private research centers.
L'archive ouverte pluridisciplinaire HAL, est destinée au dépôt et à la diffusion de documents scientifiques de niveau recherche, publiés ou non, émanant des établissements d'enseignement et de recherche français ou étrangers, des laboratoires publics ou privés. 


\section{Numerical Instability of Classical Free-Interface Component Mode Synthesis Techniques}

\author{
Ronnie Bladh, ${ }^{*}$ Christophe Pierre, ${ }^{\dagger}$ \\ and Matthew P. Castanier \\ University of Michigan, Ann Arbor, Michigan 48109-2125
}

\begin{tabular}{lll} 
& \multicolumn{1}{c}{ Nomenclature } \\
$\boldsymbol{f}_{\Gamma}$ & $=$ interface force vector \\
$\boldsymbol{G}$ & $=$ flexibility matrix \\
$\boldsymbol{G}^{r}, \boldsymbol{H}^{r}$ & $=$ residual flexibility matrix, residual inertia matrix \\
$\boldsymbol{K}, \boldsymbol{M}$ & $=$ stiffness matrix, mass matrix \\
$\boldsymbol{p}$ & $=$ generalized (modal) coordinates \\
$\boldsymbol{x}$ & $=$ physical (finite element) coordinates \\
$\boldsymbol{\kappa}, \boldsymbol{\mu}$ & $=$ condensed stiffness matrix, condensed mass matrix \\
$\boldsymbol{\Lambda}$ & $=$ diagonal matrix of eigenvalues of the kept \\
$\boldsymbol{\Phi}$ & $=$ component normal modes \\
$\boldsymbol{\Psi}$ & $=$ set of kept component normal modes \\
$\boldsymbol{\Psi}_{\Gamma}^{r, 1}, \boldsymbol{\Psi}_{\Gamma}^{r, 2}=$ & first-order and second-order residual deflections \\
$\omega$ & & of the interface degrees of freedom (DOF) \\
$\omega$ & $=$ & frequency
\end{tabular}

Subscripts

$k=$ coordinates of the kept component normal modes

$r \quad=$ coordinates of the residual flexibility modes

$\Gamma \quad=$ finite element DOF of the component interface

$\Delta=$ finite element DOF of the component interior

Superscripts

$\alpha, \beta=\quad$ the corresponding component

\section{Introduction}

$\mathbf{F}$ INITE element based component mode synthesis ${ }^{1}$ (CMS) techniques are popular tools used in structural dynamics applications. In CMS, the original structure is subdivided into smaller substructures, or components, for which normal modes are computed independently and more inexpensively. The assembled system is then represented by a truncated set of component modes through necessary compatibility constraints applied in a systematic fashion. This process yields reduced-order models based on parent finite element models of arbitrary complexity. CMS techniques are usually characterized by the manner in which the component normal modes are computed: 1) with fixed interfaces or 2) with free interfaces.

\footnotetext{
*Visiting Research Investigator, Department of Mechanical Engineering; currently Development Engineer, ALSTOM Power, 61282 Finspang, Sweden.

${ }^{\dagger}$ Professor, Department of Mechanical Engineering; pierre@ umich.edu. Senior Member AIAA.

*Associate Research Scientist, Department of Mechanical Engineering, 2279 G. G. Brown Building; mpc@umich.edu. Senior Member AIAA.
}

In general, the classical CMS methods perform very well, yielding accurate reduced-order models. For free-interface $\mathrm{CMS},{ }^{2}$ the successful methods are those that account for the effects of neglected (residual) modes, such as the methods formulated by Rubin ${ }^{3}$ and by Craig and Chang. ${ }^{4}$ However, it is shown in this study that free-interface CMS methods incorporating residual effects do have built-in numerical instability. This is due to matrix ill-conditioning that occurs when there are only very small residual contributions, for example, for large numbers of retained component modes. This numerical instability appears abruptly and can have a devastating effect on the accuracy of these methods because any likeness to the behavior of the parent finite element model vanishes. Moreover, there are no means of determining the onset of these problems a priori. As far as the authors are aware, this limitation of the classical free-interface CMS methods has not been documented in the open literature.

In all fairness, note that the reported numerical instability is unlikely to be of concern for most engineering applications because typically only a small fraction of the total number of component modes is retained. Furthermore, this instability does not exist for fixed-interface CMS methods, which are not considered here. However, for certain special applications of free-interfaceCMS methods, it may be critically important to be aware of this numerical instability. One such special case was encountered by the authors in their line of research on the modeling of mistuned bladed disks using secondary modal analysis reduction techniques. 5 This type of modeling technique involves a two-step approach: First, CMS is used to isolate blade properties for direct perturbations (blade mistuning). Second, a modal analysis is performed on the CMS model to generate a small reduced-ordermodel based on the global modes in a frequency range of interest. In this case, the primary purpose of using CMS is to cast the original finite element model in a form that is better suited for input of blade mistuning, rather than to achieve model reduction. Therefore, for simpler academic models used in parameter studies, where high model accuracy is desired, the number of retained component modes may well surpass the unknown stability limit.

In this work, two classical free-interface CMS methods ${ }^{3,4}$ are briefly reviewed, and the sources of numerical instability are highlighted. In addition, a modest modification to the classical freeinterface CMS formulation by Craig and $\mathrm{Chang}^{4}$ is presented, which alleviates the numerical instability suffered by the original method. To support the discussion, the numerical instability is demonstrated with a simple three-dimensional, two-component finite element model.

\section{Sources of Numerical Instability in Classical Free-Interface CMS}

\section{Craig-Chang Method ${ }^{4}$}

The Craig-Chang $\left(\mathrm{C}^{2}\right)$ method $^{4}$ uses as a component modal basis a truncated set of kept component normal modes of vibration, where all of the degrees of freedom (DOF) on interfaces with adjacent components are free. A complete set of residual attachment modes are then used to supplement the normal modes. The residual attachment modes represent purely computational shapes induced in the residual structure (i.e., after removing the flexibility represented by the kept normal modes) by successively applied unit loads on each interface DOF, with all other interface DOF free and unloaded. If the 
unconstrained finite element DOF of the component are partitioned into interior and interface DOF, this results in a transformation from physical (finite element) coordinates to $\mathrm{C}^{2}$ generalized (modal) coordinates as

$$
\boldsymbol{x}=\left\{\begin{array}{l}
\boldsymbol{x}_{\Delta} \\
\boldsymbol{x}_{\Gamma}
\end{array}\right\}=\left[\begin{array}{ll}
\boldsymbol{\Phi}_{\Delta} & \boldsymbol{\Psi}_{\Delta} \\
\boldsymbol{\Phi}_{\Gamma} & \boldsymbol{\Psi}_{\Gamma}
\end{array}\right]\left\{\begin{array}{l}
p_{k} \\
p_{r}
\end{array}\right\}=\boldsymbol{U p}
$$

In computing the residual attachmentmodes, one must distinguish between constrained components and unconstrained components because the latter exhibit rigid-body modes. However, the special treatment of unconstrained components is left out for brevity. (See Craig $^{1}$ for coverage of this topic.) For a constrained component, the flexibility matrix is simply the inverse of its stiffness matrix, $\boldsymbol{G}=\boldsymbol{K}^{-1}$, and the residual flexibility matrix is formed as

$$
\boldsymbol{G}^{r}=\left[\begin{array}{ll}
\boldsymbol{G}_{\Delta \Delta}^{r} & \boldsymbol{G}_{\Delta \Gamma}^{r} \\
\boldsymbol{G}_{\Delta \Gamma}^{r^{T}} & \boldsymbol{G}_{\Gamma \Gamma}^{r}
\end{array}\right]=\boldsymbol{G}-\boldsymbol{G}^{k}=\boldsymbol{G}-\boldsymbol{\Phi} \boldsymbol{\Lambda}^{-1} \boldsymbol{\Phi}^{T}
$$

Note that in the limiting case where all normal modes are retained, $\boldsymbol{G}^{r}$ will be a null matrix. However, as will be shown later, the decreasing norm of $\boldsymbol{G}^{r}$ with an increasing number of kept modes gives rise to numerical problems before the limiting case is reached.

Because the $\mathrm{C}^{2}$ method uses successive unit loads on the interface DOF to form the residual attachment modes, these mode shapes are simply the columns of $\boldsymbol{G}^{r}$ that correspond to the interface DOF:

$$
\boldsymbol{\Psi}=\left[\begin{array}{l}
\boldsymbol{\Psi}_{\Delta} \\
\boldsymbol{\Psi}_{\Gamma}
\end{array}\right]=\left[\begin{array}{l}
\boldsymbol{G}_{\Delta \Gamma}^{r} \\
\boldsymbol{G}_{\Gamma \Gamma}^{r}
\end{array}\right]
$$

With this modal basis, the condensed $\mathrm{C}^{2}$ mass and stiffness matrices for each component become

$$
\boldsymbol{\mu}=\left[\begin{array}{cc}
\boldsymbol{I} & \mathbf{0} \\
\mathbf{0} & \boldsymbol{\Psi}^{T} \boldsymbol{M} \boldsymbol{\Psi}
\end{array}\right], \quad \boldsymbol{\kappa}=\left[\begin{array}{cc}
\boldsymbol{\Lambda} & \mathbf{0} \\
\mathbf{0} & \boldsymbol{\Psi}_{\Gamma}
\end{array}\right], \quad \boldsymbol{p}=\left\{\begin{array}{l}
\boldsymbol{p}_{k} \\
\boldsymbol{p}_{r}
\end{array}\right\}
$$

The assembly of two components, $\alpha$ and $\beta$, is achieved by satisfying force equilibrium (Craig, ${ }^{1}$ p. 488) and displacement compatibility over the component interfaces, which, after simplification, results in the constraints

$$
\begin{gathered}
\boldsymbol{p}_{r}^{\beta}=-\boldsymbol{p}_{r}^{\alpha}=\boldsymbol{p}_{r} \\
\boldsymbol{\Phi}_{\Gamma}^{\alpha} \boldsymbol{p}_{k}^{\alpha}-\boldsymbol{\Phi}_{\Gamma}^{\beta} \boldsymbol{p}_{k}^{\beta}+\left[\boldsymbol{\Psi}_{\Gamma}^{\alpha}+\boldsymbol{\Psi}_{\Gamma}^{\beta}\right] \boldsymbol{p}_{r}=\mathbf{0}
\end{gathered}
$$

These constraintequations lead to the synthesized $C^{2}$ representation of a two-component structure:

$$
\begin{gathered}
\boldsymbol{M}^{\mathrm{C}^{2}}=\left[\begin{array}{cc}
\boldsymbol{I}+\boldsymbol{\Phi}_{\Gamma}^{\alpha^{T}} \boldsymbol{M}_{1} \boldsymbol{\Phi}_{\Gamma}^{\alpha} & -\boldsymbol{\Phi}_{\Gamma}^{\alpha^{T}} \boldsymbol{M}_{1} \boldsymbol{\Phi}_{\Gamma}^{\beta} \\
-\boldsymbol{\Phi}_{\Gamma}^{\beta^{T}} \boldsymbol{M}_{1}^{T} \boldsymbol{\Phi}_{\Gamma}^{\alpha} & \boldsymbol{I}+\boldsymbol{\Phi}_{\Gamma}^{\beta^{T}} \boldsymbol{M}_{1} \boldsymbol{\Phi}_{\Gamma}^{\beta}
\end{array}\right] \\
\boldsymbol{K}^{\mathrm{C}^{2}}=\left[\begin{array}{cc}
\boldsymbol{\Lambda}^{\alpha}+\boldsymbol{\Phi}_{\Gamma}^{\alpha^{T}} \boldsymbol{K}_{1} \boldsymbol{\Phi}_{\Gamma}^{\alpha} & -\boldsymbol{\Phi}_{\Gamma}^{\alpha^{T}} \boldsymbol{K}_{1} \boldsymbol{\Phi}_{\Gamma}^{\beta} \\
-\boldsymbol{\Phi}_{\Gamma}^{\beta^{T}} \boldsymbol{K}_{1}^{T} \boldsymbol{\Phi}_{\Gamma}^{\alpha} & \boldsymbol{\Lambda}^{\beta}+\boldsymbol{\Phi}_{\Gamma}^{\beta^{T}} \boldsymbol{K}_{1} \boldsymbol{\Phi}_{\Gamma}^{\beta}
\end{array}\right], \quad \boldsymbol{p}^{\mathrm{C}^{2}}=\left\{\begin{array}{l}
\boldsymbol{p}_{k}^{\alpha} \\
\boldsymbol{p}_{k}^{\beta}
\end{array}\right\}
\end{gathered}
$$

where

$$
\begin{gathered}
\boldsymbol{K}_{1}=\left[\boldsymbol{\Psi}_{\Gamma}^{\alpha}+\boldsymbol{\Psi}_{\Gamma}^{\beta}\right]^{-1} \\
\boldsymbol{M}_{1}=\boldsymbol{K}_{1}\left[\boldsymbol{\Psi}^{\alpha^{T}} \boldsymbol{M}^{\alpha} \boldsymbol{\Psi}^{\alpha}+\boldsymbol{\Psi}^{\beta^{T}} \boldsymbol{M}^{\beta} \boldsymbol{\Psi}^{\beta}\right] \boldsymbol{K}_{1}
\end{gathered}
$$

Note that only the generalized coordinates pertaining to the kept component normal modes of vibration remain in the final $\mathrm{C}^{2}$ representation. This makes the $\mathrm{C}^{2}$ model particularly compact and, therefore, exceptionally efficient.

In the $\mathrm{C}^{2}$ method, the numerical instability is introduced by solving for $\boldsymbol{p}_{r}$ in Eq. (6). This assumes that the inverse of $\left[\boldsymbol{\Psi}_{\Gamma}^{\alpha}+\boldsymbol{\Psi}_{\Gamma}^{\beta}\right]=\left[\boldsymbol{G}_{\Gamma \Gamma}^{r, \alpha}+\boldsymbol{G}_{\Gamma \Gamma}^{r, \beta}\right]$ exists and, thus, that the conditioning of the summed interface residual flexibilities is sufficient. Although this assumption is likely to be adequate for most engineering applications, it will not be true when the numbers of kept component normal modes become sufficiently large because the residual flexibility partitions will approach null matrices.

\section{Rubin Method ${ }^{3}$}

As in the $\mathrm{C}^{2}$ method, the Rubin (R) method ${ }^{3}$ uses a truncated set of kept component normal modes of vibration as a componentmodal basis, where all of the interface DOF are free. However, there are distinct differences between the two methods, some of which are summarized as follows. The $\mathrm{C}^{2}$ method uses residual attachment modes induced by a complete set of successive unit loads on the interfaceDOF, and it follows a Rayleigh-Ritz procedure. In contrast, the $\mathrm{R}$ method retains the physical interface DOF to solve for an arbitrary force on the component interface, and it does not follow a Rayleigh-Ritz procedure. The $\mathrm{R}$ technique allows for inclusion of residual inertia by the use of a second-order Maclaurin series expansion when solving for the interface force.

With forces acting on the componentinterfaceDOF only, the firstorder (static) residual deflections of the interface DOF are obtained as

$$
\Psi_{\Gamma}^{r, 1}=\boldsymbol{G}_{\Gamma \Gamma}^{r} f_{\Gamma}
$$

where the definition of the residual flexibility matrix $\boldsymbol{G}^{r}$ follows the $\mathrm{C}^{2}$ approach exactly. By the use of Eq. (8), and the assumption of harmonic motion, the second-order residual deflections of the interface DOF are obtained as

$$
\boldsymbol{\Psi}_{\Gamma}^{r, 2}=\boldsymbol{G}_{\Gamma \Gamma}^{r}\left[\boldsymbol{f}_{\Gamma}-\left(-\omega^{2} \boldsymbol{M} \boldsymbol{\Psi}_{\Gamma}^{r, 1}\right)\right]=\left[\boldsymbol{G}_{\Gamma \Gamma}^{r}+\omega^{2} \boldsymbol{H}_{\Gamma \Gamma}^{r}\right] \boldsymbol{f}_{\Gamma}
$$

where $\boldsymbol{H}_{\Gamma \Gamma}^{r}$ is the interface partition of the residual inertia matrix, which is computed as

$$
\boldsymbol{H}^{r}=\left[\begin{array}{cc}
\boldsymbol{H}_{\Delta \Delta}^{r} & \boldsymbol{H}_{\Delta \Gamma}^{r} \\
\boldsymbol{H}_{\Delta \Gamma}^{r^{T}} & \boldsymbol{H}_{\Gamma \Gamma}^{r}
\end{array}\right]=\boldsymbol{G}^{r} \boldsymbol{M} \boldsymbol{G}^{r}
$$

by virtue of orthogonality between kept and residual flexibility with respect to the mass matrix, that is, $\boldsymbol{G}^{k} \boldsymbol{M} \boldsymbol{G}^{r} \equiv \mathbf{0}$.

The physical displacements of the interface DOF can now be represented as a superposition of the kept component normal modes and the second-order residual deflections from Eq. (9):

$$
\boldsymbol{x}_{\Gamma}=\boldsymbol{\Phi}_{\Gamma} \boldsymbol{p}_{k}+\boldsymbol{\Psi}_{\Gamma}^{r, 2}=\boldsymbol{\Phi}_{\Gamma} \boldsymbol{p}_{k}+\left[\boldsymbol{G}_{\Gamma \Gamma}^{r}+\omega^{2} \boldsymbol{H}_{\Gamma \Gamma}^{r}\right] \boldsymbol{f}_{\Gamma}
$$

Then, with a second-order Maclaurin series expansion in $\omega$, the interface force may be solved from Eq. (11) as

$$
\boldsymbol{f}_{\Gamma} \approx\left[\boldsymbol{K}_{\Gamma \Gamma}^{r}-\omega^{2} \boldsymbol{M}_{\Gamma \Gamma}^{r}\right]\left[\boldsymbol{x}_{\Gamma}-\boldsymbol{\Phi}_{\Gamma} \boldsymbol{p}_{k}\right]
$$

where

$$
\boldsymbol{K}_{\Gamma \Gamma}^{r}=\boldsymbol{G}_{\Gamma \Gamma}^{r^{-1}}, \quad \boldsymbol{M}_{\Gamma \Gamma}^{r}=\boldsymbol{G}_{\Gamma \Gamma}^{r^{-1}} \boldsymbol{H}_{\Gamma \Gamma}^{r} \boldsymbol{G}_{\Gamma \Gamma}^{r^{-1}}
$$

In addition, the kept modes must satisfy dynamic equilibrium:

$$
\left[\boldsymbol{\Lambda}-\omega^{2} \boldsymbol{I}\right] \boldsymbol{p}_{k}=\boldsymbol{\Phi}_{\Gamma}^{T} \boldsymbol{f}_{\Gamma}
$$

Identification of mass $\left(\omega^{2}\right)$ and stiffness $\left(\omega^{0}\right)$ terms in Eqs. (12) and (13) yields the condensed $\mathrm{R}$ mass and stiffness matrices for each component as

$\boldsymbol{\mu}=\left[\begin{array}{ll}\boldsymbol{\mu}_{k k} & \boldsymbol{\mu}_{k \Gamma} \\ \boldsymbol{\mu}_{k \Gamma}^{T} & \boldsymbol{\mu}_{\Gamma \Gamma}\end{array}\right], \quad \boldsymbol{\kappa}=\left[\begin{array}{cc}\boldsymbol{\kappa}_{k k} & \boldsymbol{\kappa}_{k \Gamma} \\ \boldsymbol{\kappa}_{k \Gamma}^{T} & \boldsymbol{\kappa}_{\Gamma \Gamma}\end{array}\right], \quad \boldsymbol{p}=\left\{\begin{array}{l}\boldsymbol{p}_{k} \\ \boldsymbol{x}_{\Gamma}\end{array}\right\}$

where

$$
\begin{array}{ll}
\boldsymbol{\mu}_{k k}=\boldsymbol{I}+\boldsymbol{\Phi}_{\Gamma}^{T} \boldsymbol{M}_{\Gamma \Gamma}^{r} \boldsymbol{\Phi}_{\Gamma} & \boldsymbol{\kappa}_{k k}=\boldsymbol{\Lambda}+\boldsymbol{\Phi}_{\Gamma}^{T} \boldsymbol{K}_{\Gamma \Gamma}^{r} \boldsymbol{\Phi}_{\Gamma} \\
\boldsymbol{\mu}_{k \Gamma}=-\boldsymbol{\Phi}_{\Gamma}^{T} \boldsymbol{M}_{\Gamma \Gamma}^{r} & \boldsymbol{\kappa}_{k \Gamma}=-\boldsymbol{\Phi}_{\Gamma}^{T} \boldsymbol{K}_{\Gamma \Gamma}^{r} \\
\boldsymbol{\mu}_{\Gamma \Gamma}=\boldsymbol{M}_{\Gamma \Gamma}^{r} & \boldsymbol{\kappa}_{\Gamma \Gamma}=\boldsymbol{K}_{\Gamma \Gamma}^{r}
\end{array}
$$


Again, the model assembly is achieved by satisfaction of the displacement compatibility and internal force equilibrium over the component interfaces, which, after simplification, results in the constraints

$x_{\Gamma}^{\alpha}=x_{\Gamma}^{\beta}=x_{\Gamma}$

$\left[\boldsymbol{\kappa}_{k \Gamma}^{\alpha^{T}}-\omega^{2} \boldsymbol{\mu}_{k \Gamma}^{\alpha^{T}}\right] \boldsymbol{p}_{k}^{\alpha}+\left[\boldsymbol{\kappa}_{\Gamma \Gamma}^{\alpha}+\boldsymbol{\kappa}_{\Gamma \Gamma}^{\beta}-\omega^{2}\left(\boldsymbol{\mu}_{\Gamma \Gamma}^{\alpha}+\boldsymbol{\mu}_{\Gamma \Gamma}^{\beta}\right)\right] \boldsymbol{x}_{\Gamma}$

$$
+\left[\boldsymbol{\kappa}_{k \Gamma}^{\beta^{T}}-\omega^{2} \boldsymbol{\mu}_{k \Gamma}^{\beta^{T}}\right] \boldsymbol{p}_{k}^{\beta}=\mathbf{0}
$$

These constraint equations lead to the synthesized $\mathrm{R}$ representation of a two-component structure:

$$
\begin{gathered}
\boldsymbol{M}^{\mathrm{R}}=\left[\begin{array}{ccc}
\boldsymbol{\mu}_{k k}^{\alpha} & \boldsymbol{\mu}_{k \Gamma}^{\alpha} & \mathbf{0} \\
\boldsymbol{\mu}_{k \Gamma}^{\alpha^{T}} & \boldsymbol{\mu}_{\Gamma \Gamma}^{\alpha}+\boldsymbol{\mu}_{\Gamma \Gamma}^{\beta} & \boldsymbol{\mu}_{k \Gamma}^{\beta^{T}} \\
\mathbf{0} & \boldsymbol{\mu}_{k \Gamma}^{\beta} & \boldsymbol{\mu}_{k k}^{\beta}
\end{array}\right] \\
\boldsymbol{K}^{\mathrm{R}}=\left[\begin{array}{ccc}
\boldsymbol{\kappa}_{k k}^{\alpha} & \boldsymbol{\kappa}_{k \Gamma}^{\alpha} & \mathbf{0} \\
\boldsymbol{\kappa}_{k \Gamma}^{\alpha} & \boldsymbol{\kappa}_{\Gamma \Gamma}^{\alpha}+\boldsymbol{\kappa}_{\Gamma \Gamma}^{\beta} & \boldsymbol{\kappa}_{k \Gamma}^{\beta^{T}} \\
\mathbf{0} & \boldsymbol{\kappa}_{k \Gamma}^{\beta} & \boldsymbol{\kappa}_{k k}^{\beta}
\end{array}\right],
\end{gathered}
$$

On comparison of this model with the $C^{2}$ model of Eq. (7), it is seen that the $\mathrm{R}$ model has the additional expense of retained physical interface DOF.

In the $\mathrm{R}$ method, the numerical instability is introduced by solving for the interface force vector by the second-order Maclaurin series expansion in Eq. (12), which requires the inverse of each component's interface residual flexibility $\boldsymbol{G}_{\Gamma \Gamma}^{r^{-1}}$.

\section{Stabilized Free-Interface CMS Method}

The proposed stabilized free-interface CMS method is simply an alternate formulation of the previously derived $\mathrm{C}^{2}$ method, and, therefore, it will be referred to as the stabilized Craig-Chang $\left(\mathrm{SC}^{2}\right)$ method. As noted earlier, the displacementcompatibility constraints of Eq. (6) are used to eliminate the generalized coordinates $\boldsymbol{p}_{r}$ before the $\mathrm{C}^{2}$ system equations are formulated. This step leads to the particularly compact $\mathrm{C}^{2} \mathrm{CMS}$ model, but it also requires inverting the matrix of the summed componentinterface residual flexibilities, which introduces the possibility of numerical instability. Hence, this matrix inversion must be avoided to yield an unconditionallynumerically stable formulation. Therefore, the $\mathrm{SC}^{2}$ method is formulated by retention of the generalized coordinates $\boldsymbol{p}_{r}$ and the enforcement of displacement compatibility by the inclusion of Eq. (6) in the system equations. This yields the synthesized $\mathrm{SC}^{2}$ representation of a two-component structure, as follows:

$$
\begin{gathered}
\boldsymbol{M}^{\mathrm{SC}^{2}}=\left[\begin{array}{ccc}
\boldsymbol{I} & \mathbf{0} & \mathbf{0} \\
\mathbf{0} & \mathbf{0} & \mathbf{0} \\
\mathbf{0} & \mathbf{0} & \boldsymbol{I}
\end{array}\right], \\
\boldsymbol{K}^{\mathrm{SC}^{2}}=\left[\begin{array}{ccc}
\boldsymbol{\Lambda}^{\alpha} & \boldsymbol{\Phi}_{\Gamma}^{\alpha^{T}} & \mathbf{0} \\
\boldsymbol{\Phi}_{\Gamma}^{\alpha} & \boldsymbol{\Psi}_{\Gamma}^{\alpha}+\boldsymbol{\Psi}_{\Gamma}^{\beta} & -\boldsymbol{\Phi}_{\Gamma}^{\beta} \\
\mathbf{0} & -\boldsymbol{\Phi}_{\Gamma}^{\beta^{T}} & \boldsymbol{\Lambda}^{\beta}
\end{array}\right] \\
\boldsymbol{p}^{\mathrm{SC}^{2}}=\left\{\begin{array}{l}
\boldsymbol{p}_{k}^{\alpha} \\
\boldsymbol{p}_{r} \\
\boldsymbol{p}_{k}^{\beta}
\end{array}\right\}
\end{gathered}
$$

Note that the generalized coordinates $\boldsymbol{p}_{r}$ pertain to the complete set of residual attachment modes and, thus, are equal in number to the physical interface DOF. Hence, the stabilization of the classical $C^{2}$ formulation is achieved at the expense of a substantial increase in model size. This is clearly undesirable, and it is, therefore, proposed that the $\mathrm{SC}^{2}$ approach may serve as a $\mathrm{C}^{2}$ model quality assessment tool. The $\mathrm{C}^{2}$ model may be checked against the unconditionally stable $\mathrm{SC}^{2}$ model for consistency during the CMS model construction, before proceeding to comprehensive dynamic analyses using the more computationally efficient $\mathrm{C}^{2}$ model. Because the $\mathrm{SC}^{2}$ model is based on exactly the same component modal quantities as the $\mathrm{C}^{2}$ model, such consistency checks can be made relatively easily.

As a final remark, note that the $\mathrm{R}$ method can not be stabilized because the inversion of residual flexibilities is inherent to solving for the interface force by the second-order Maclaurin series expansion in Eq. (12).

\section{Modal Convergence and Numerical Stability Issues}

Here, the numerical instability exhibited by the classical freeinterface CMS techniques is demonstrated with the simple twocomponent test model (Fig. 1). The model is constructed from second-orderbrick elements (20-noded solids) and has a total of 651 unconstrained DOF. The numerical stability issues are explored by means of modal convergencetrends for the free-interfaceCMS techniques. (Finite element analyses were performed with NASTRAN, and CMS computations were performed with MATLAB ${ }^{\circledR}$. Double precision was used for all computations.) The employed convergence measure is the average error of the CMS results, relative to the finite element values, for the four lowest natural frequencies of the test model. The deformed finite element meshes for these four modes are shown in Fig. 1.

The modal convergencetrends are shown in Fig. 2. Note the quick convergence shown by the classical free-interface CMS methods: The $\mathrm{C}^{2}$ and $\mathrm{R}$ techniques yield highly accurate reduced-order models with very few kept component normal modes. However, beyond certain numbers of kept modes, both techniques exhibit sudden onsets of dramatic deteriorationin model quality. Beyond these points, approximately 450 modes for $\mathrm{C}^{2}$ and 300 modes for $\mathrm{R}$, natural frequencies and mode shapes bear little or no resemblance with the reference solutions. In contrast, although sufficiently accurate for most applications, the $\mathrm{SC}^{2}$ model displays much slower modal convergence than the classical free-interface CMS techniques. On the

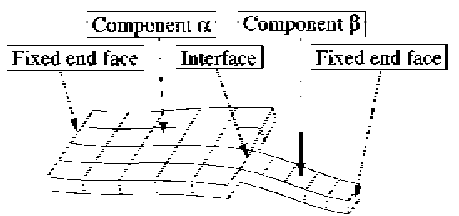

a)

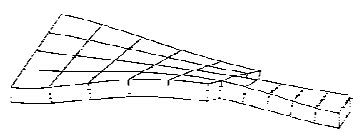

b)

Fig. 1 Deformed finite element meshes for the four lowest modes of the two-component test model: a) mode $1,103.6 \mathrm{~Hz}$; b) mode $2,172.8 \mathrm{~Hz}$; c) mode $3,341.4 \mathrm{~Hz}$; and d) mode $4,420.6 \mathrm{~Hz}$.

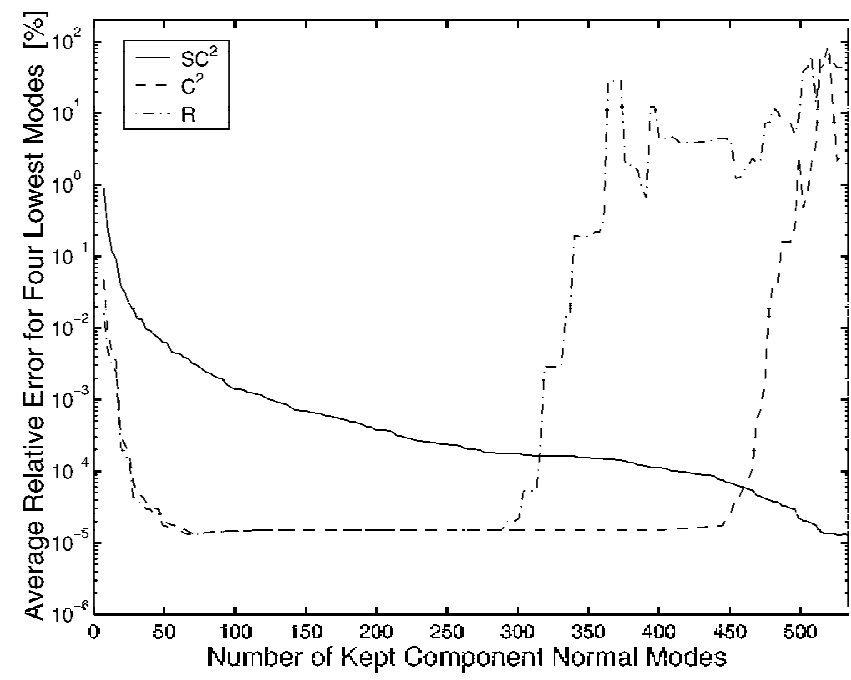

Fig. 2 Convergence of the four lowest natural frequencies for the considered free-interface CMS methods relative to finite element results. 


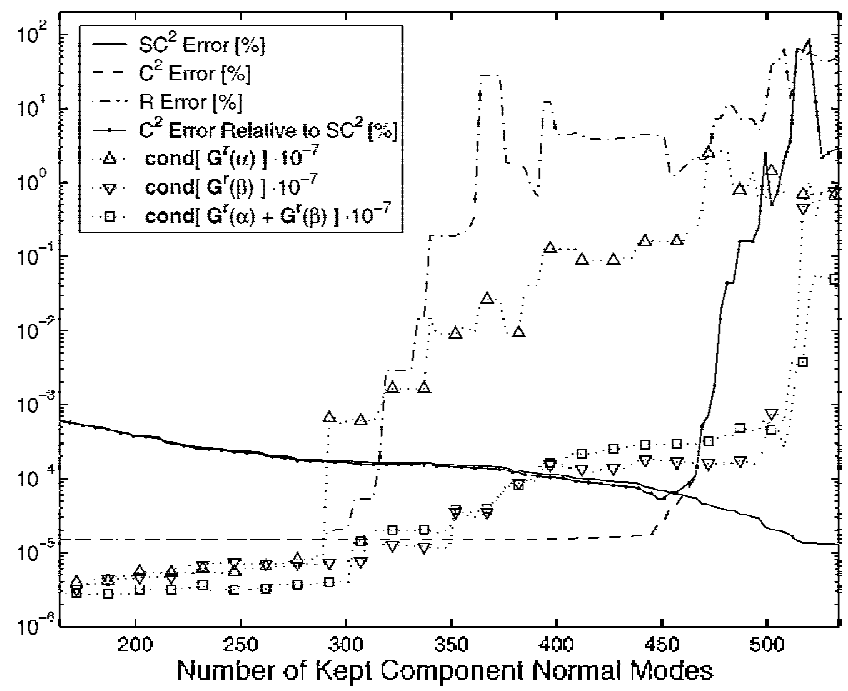

Fig. 3 Effect of component interface residual flexibility matrix conditioning on free-interface CMS model behavior.

other hand, the $\mathrm{SC}^{2}$ model is unconditionally stable by construction. As the number of kept component normal modes increases, the steady improvement in accuracy continues for the $\mathrm{SC}^{2}$ model until all such modes are included.

As discussed earlier, the onset of the numerical instability for the classical free-interface CMS methods is caused by the inversion of residual flexibility matrix partitions, either separate $(\mathrm{R})$ or summed $\left(C^{2}\right)$. These matrices become progressivelymore ill conditioned as the number of kept normal modes (i. e., the kept flexibility) increases, and they will, in theory, eventually become null matrices. Figure 3 illustrates the correlation between the observed numerical instability and the conditioning of interface residual flexibility matrices, cond $\left[\boldsymbol{G}^{r}\right]$ (subscript $\Gamma \Gamma$ is dropped). The onset of numerical instability for the $\mathrm{R}$ model at just over $300 \mathrm{kept}$ modes is clearly marked by a degrading shift of nearly two orders of magnitude in the conditioning of the interface residual flexibility matrix for component $\alpha$. The impact of interface residual flexibility matrix conditioning is less apparent for the $\mathrm{C}^{2}$ model. However, the later onset of instability exhibited by the $\mathrm{C}^{2}$ model is explained by the inversion of the summed residual flexibility matrices, as opposed to the separate inversions of componentwise matrices of the R model. An additional note, based on other models used by the authors, is that the onset of numerical instability can occur at significantly lower fractions of the total number of component normal modes, for example, less than $40 \%$, compared to the results for the particular test model used in this study.

Figure 3 also illustrates the use of the $\mathrm{SC}^{2}$ method as an inexpensive $\mathrm{C}^{2}$ model quality assessment tool. The plotted $\mathrm{C}^{2}$ error relative to $\mathrm{SC}^{2}$ in Fig. 3 is the same four-mode average error considered elsewhere in this section, but with the $\mathrm{SC}^{2}$ results used as reference values. As shown in Fig. 3, this relative error measure provides very clear information on the quality of the $\mathrm{C}^{2}$ model.

\section{Conclusions}

Clearly, the found numerical instability of the classical freeinterface CMS methods is of little practical importance for most engineering applications. Unless a large fraction of the total number of component normal modes is retained, this instability is not likely to present a problem. However, special cases exist where this numerical instability can become a factor. In such cases, awareness of this limitation of classical free-interface CMS methods may be of critical importance.

\section{References}

${ }^{1}$ Craig, R. R., Structural Dynamics: An Introduction to Computer Methods, Wiley, New York, 1981, Chap. 19.

${ }^{2}$ Craig, R. R., and Chang, C.-J., "On the Use of Attachment Modes in Substructure Coupling for Dynamic Analysis," Proceedings of the 18th AIAA/ASME/ASCE/AHS/ASC Structures, Structural Dynamics, and Materials Conference, AIAA, New York, 1977, pp. 89-99.

${ }^{3}$ Rubin, S., "Improved Component-Mode Representation for Structural Dynamic Analysis," AIAA Journal, Vol. 13, No. 8, 1975, pp. 995-1006.

${ }^{4}$ Craig, R. R., and Chang, C.-J., "Free-Interface Methods of Substructure Coupling for Dynamic Analysis," AIAA Journal, Vol. 14, No. 11, 1976 , pp. $1633-1635$.

${ }^{5}$ Bladh, R., Castanier, M. P., and Pierre, C., "Component-Mode-Based Reduced Order Modeling Techniques for Mistuned Bladed Disks-Part I: Theoretical Models," Journal of Engineering for Gas Turbines and Power, Vol. 123, No. 1, 2001, pp. 89-99. 